\title{
The Role of Overshoot in Solar Activity: A Direct Simulation of the Dynamo
}

\author{
A. Brandenburg ${ }^{1,2}$, R.L. Jennings ${ }^{3}, \AA$. Nordlund ${ }^{4}$, \\ R.F. Stein ${ }^{5}$, I. Tuominen ${ }^{1}$ \\ ${ }^{1}$ Observatory and Astrophysics Laboratory, University of Helsinki, Finland \\ ${ }^{2}$ NORDITA, Blegdamsvej 17, DK-2100 Copenhagen, Denmark \\ ${ }^{3}$ Dept. of Appl. Math. and Theor. Physics, Cambridge CB3 9EW, UK \\ ${ }^{4}$ Copenhagen University Observatory, DK-1350 Copenhagen K, Denmark \\ ${ }^{5}$ Dept. of Physics and Astronomy, Michigan State University, USA
}

\begin{abstract}
We investigate convective overshoot in a layer of electrically conducting fluid. The radiative conductivity is assumed to be larger in the lower part of the layer which makes it stable to convective motions, yet penetrative convection from the upper layer can occur. The numerical resolution is $63^{3}$ gridpoints. We observe a dynamo effect for magnetic Reynolds numbers around one thousand when a magnetic seed field is rapidly concentrated to form flux tubes. Later the average magnetic field is expelled from the convectively unstable regions, but it accumulates in the interface between the convection zone and the radiative interior.
\end{abstract}

\section{Introduction}

The nature of the solar dynamo is unclear. Does the dynamo operate in the entire convection zone, or in the overshoot layer beneath? Is magnetic buoyancy a "problem" for the dynamo? Is the solar dynamo fast or slow?

We investigate these questions using a direct simulation of turbulent hydromagnetic convection. The presence of a stably stratified overshoot layer is modelled by assuming the radiative diffusivity in the lower half of the simulated domain to be larger than that in the upper unstably stratified part (cf. Hurlburt et al., 1986). 


\section{The basic equations}

We solve the induction equation and the equations for conservation of momentum, energy, and mass

$$
\begin{gathered}
\frac{\partial \boldsymbol{B}}{\partial t}=\operatorname{curl}(\boldsymbol{u} \times \boldsymbol{B})+\eta \nabla^{2} \boldsymbol{B}, \\
\frac{\mathrm{D} \boldsymbol{u}}{\mathrm{D} t}=-\frac{1}{\rho} \nabla p+\boldsymbol{g}-2 \boldsymbol{\Omega} \times \boldsymbol{u}+\frac{1}{\rho} \boldsymbol{J} \times \boldsymbol{B}+\frac{1}{\rho} \operatorname{Div} \boldsymbol{\tau}, \\
\frac{\mathrm{D} e}{\mathrm{D} t}=-\frac{p}{\rho} \operatorname{div} \boldsymbol{u}+\frac{1}{\rho} \nabla(\mathcal{K} \nabla e)+\tau_{i j} u_{i, j}+\frac{\eta}{\rho} \boldsymbol{J}^{2} \\
\frac{\mathrm{D} \ln \rho}{\mathrm{D} t}=-\operatorname{div} \boldsymbol{u},
\end{gathered}
$$

For details of the notation see Paper I (Brandenburg et al., 1990). We assume the upper and lower boundary to be a stress-free perfect conductor. A constant radiative flux is imposed at the bottom and the surface temperature is kept constant. In the current implementation of the code one time step takes for $63^{3}$ grid points about $3.4 \mathrm{sec}$ of CPU time. With the four processors on the Cray-XMP/432 the real system time can be of the order of only one second per time step.

The governing nondimensional parameters in our simulation are a Taylor number of $10^{5}$ (based on the thickness of the unstable layer), a Rayleigh number of $10^{6}$ (approx. 50 times supercritical), a Prandtl number of 0.2 , and a magnetic Prandtl number $\nu / \eta=4$. The density contrast is approximately 1:10. The resulting Reynolds number is around 300 (based on rms-velocity) and the Chandrasekhar number $3 \times 10^{3}$. The Rossby number is approximately unity and the Elsasser number about ten.

\section{Results}

We find large scale coherent magnetic structures similar to vorticity tubes seen in homogeneous turbulence (She et al., 1990; Vincent and Meneguzzi, 1990). In a video animation we observed that magnetic flux tubes are pulled downwards and wounded up close to the interface between the convection zone and the radiative interior. A dynamo effect is found for magnetic Prandtl numbers larger than unity. The magnetic Reynolds numbers are in our case much higher than those for dynamo action in Bousinesq convection (Meneguzzi and Pouquet, 1989). The initial growth rate of magnetic energy is comparable with the convective turnover time and we can therefore speak of a fast dynamo.

The magnetic energy has a maximum at the interface. This is also the location where both induction effects and Ohmic dissipation are largest. The fact that there is no accumulation of magnetic flux in the upper layers leads us to believe that the perfectly conducting upper boundary is not artificially suppressing magnetic buoyancy, as suggested by Petrovay (1991) during the conference. However, 
we often observe that tubes organize themselves vertically in the upper unstably stratified part and thus escape the buoyancy mechanism to work efficiently.

We have plotted in Fig. 1 vectors of vorticity $\boldsymbol{\omega}=$ curl $\boldsymbol{u}$, electric current $\boldsymbol{J}$, and magnetic field $\boldsymbol{B}$. Vectors are plotted only where their magnitude exceeds a certain threshold.
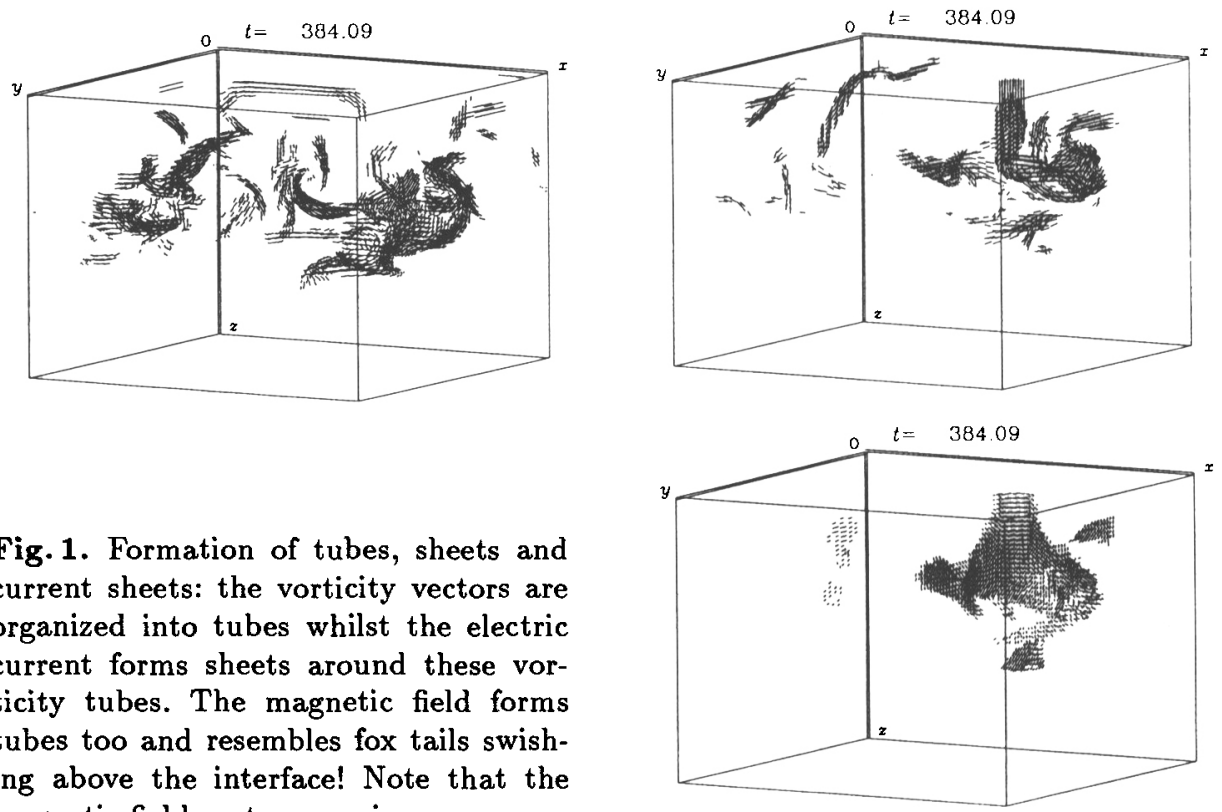

Fig. 1. Formation of tubes, sheets and current sheets: the vorticity vectors are organized into tubes whilst the electric current forms sheets around these vorticity tubes. The magnetic field forms tubes too and resembles fox tails swishing above the interface! Note that the magnetic field vectors are, in some cases, aligned with the vorticity vectors.

\section{References}

Brandenburg, A., Nordlund, Å., Pulkkinen, P., Stein, R.F., Tuominen, I.: 1990, Astron. Astrophys. 232, 277 (Paper I)

Hurlburt, N.E., Toomre, J., Massaguer, J.M.: 1986, Astrophys. J. 311, 563

Meneguzzi, M., Pouquet, A.: 1989, J. Fluid. Mech. 205, 297

Petrovay, K.: 1991, these Proceedings

She, Z.-S., Jackson, E., Orszag, S. A.: 1990, Nature 344, 226

Vincent, A., Meneguzzi, M.: 1990, J. of Fluid Mech., in press 